\title{
miR-494-3p promotes the progression of endometrial cancer by regulating the PTEN/PI3K/AKT pathway
}

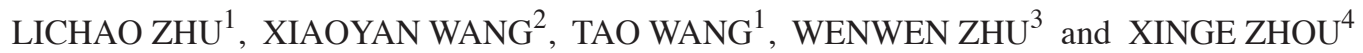 \\ ${ }^{1}$ Department of Geriatrics, The Second Affiliated Hospital of Zhengzhou University, Zhengzhou, Henan 450014; \\ ${ }^{2}$ Department of Rehabilitation, Zhangqiu Hospital of Traditional Chinese Medicine, Jinan, Shandong 250200; \\ ${ }^{3}$ Department of Stomatology, The First Affiliated Hospital of Zhengzhou University, Zhengzhou, Henan 450014; \\ ${ }^{4}$ Department of Neurosurgery, Suiping People's Hospital, Zhumadian, Henan 463100, P.R. China
}

Received March 11, 2018; Accepted October 9, 2018

DOI: $10.3892 / \mathrm{mmr} .2018 .9649$

\begin{abstract}
MicroRNAs (miRs) are essential regulators in the development and progression of cancer. The role of miR-494-3p in endometrial cancer (EC) has not yet been investigated. In the present study, the expression levels of miR-494-3p were significantly upregulated in EC tissues compared with adjacent normal tissues. Furthermore, upregulation of miR-494-3p in patients with EC indicated poorer prognosis; miR-494-3p overexpression significantly promoted the proliferation, migration and invasion of HHUA and JEC cells in vitro. Consistently, inhibition of miR-494-3p in HHUA cells significantly suppressed tumor growth in vivo in a xenograft model. Additionally, phosphatase and tensin homolog (PTEN) was revealed to be a direct target of miR-494-3p in EC cells. Furthermore, overexpression of miR-494-3p inhibited PTEN expression and consequently activated the downstream phosphoinositide 3-kinase/protein kinase B (PI3K/AKT) signialing pathway. Restoration of PTEN or inhibition of PI3K/AKT pathway also abolished miR-494-3p-mediated proliferation, migration and invasion of HHUA and JEC cells. In summary, the results of the present study revealed the importance of the miR-494-3p/PTEN/PI3K/AKT axis in the progression of EC, which may provide novel insight into potential therapeutic targets for the treatment of EC.
\end{abstract}

\section{Introduction}

Endometrial cancer (EC) is the fourth most prevalent and aggressive type of gynecological cancer, which has become a great threat against women's health $(1,2)$. Annually, >280,000

Correspondence to: Dr Lichao Zhu, Department of Geriatrics, The Second Affiliated Hospital of Zhengzhou University, 2 Jingba Road, Zhengzhou, Henan 450014, P.R. China

E-mail: zhulichao1699@163.com

Key words: microRNA-494-3p, endometrial cancer, proliferation, phosphatse and tensin homolog/phosophoinositide 3-kinase/protein kinase $\mathrm{B}$, metastasis women are diagnosed with EC worldwide; EC results in numerous cancer-associated mortalities (3). Unremitting efforts have been made to develop effective methods for treating EC in the past decades. Radical surgery may be the only effective therapeutic method for early stage EC. At present, the incidence of EC is still rising due to tumor recurrence and metastasis $(4,5)$. Therefore, investigating the molecular mechanisms of endometrial carcinogenesis, screening out effective diagnostic biomarkers and developing promising therapeutic targets are particularly important for the treatment of EC.

MicroRNAs (miRNAs/miRs) are a member of the family of noncoding RNAs and have a length of 21-23 nucleotides (6). Numerous reports have demonstrated that miRNAs could regulate gene expression by directly associating with the 3'-untranslated region of target mRNAs $(7,8)$. By modulating the protein expression levels of target genes, miRNAs are widely involved in various biological processes and human diseases (9). In addition, accumulating evidence has indicated that miRNAs are essential regulators in a variety of cancers by serving as oncogenes or tumor suppressors (10). For instance, miR-199a/b-3p suppresses the proliferation of gastric cancer cells by regulating P21 activated kinase 4/mitogen activated protein kinase/extracellular signal-regulated kinase signaling pathway (11). Li et al (12) indicated that miR-34a directly targeted high-mobility group box 1 and inhibited the proliferation, migration and invasion of cancer cells in cutaneous squamous cell carcinoma. In EC, Zhao et al (13) revealed that miR-126 inhibited the migration and invasion of EC cells by targeting insulin receptor substrate 1 . In summary, the aforementioned findings highlighted the importance of miRNAs in the progression of cancer, which suggests that miRNAs may be promising biomarkers and therapeutic targets in the treatment of cancers.

Recent studies have indicated that miR-494-3p could induce lung carcinogenesis and regulate the proliferation, invasion, migration and apoptosis of human glioblastoma cells $(14,15)$. On the contrary, it has been suggested that miR-494-3p acts as a tumor suppressor in some cancers, such as prostate (16) and lung cancer (14). For example, Shen et al (16) have reported that miR-494-3p suppresses the proliferation, invasion, and migration of prostate cancer; however, the role of 
miR-494-3p remains unknown in EC. In the present study, it was revealed that miR-494-3p was significantly upregulated in EC tissues and was associated with the prognosis of patients with EC. In addition, overexpression of miR-494-3p markedly promoted the proliferation, migration and invasion of EC cells. Furthermore, miR-494-3p directly targeted phosphatase and tensin homolog (PTEN) and consequently regulated phosphoinositide 3-kinase (PI3K)/protein kinase B (AKT) pathway in EC cells. Additionally, inhibition of the PI3K/AKT pathway abolished miR-494-3p-mediated effects on HHUA and JEC cells. In summary, the results of the present study revealed the pivotal role of the miR-494-3p/PTEN/PI3K/AKT pathway in EC progression, which may provide novel insight in the identification of therapeutic targets for the treatment of EC.

\section{Materials and methods}

Patient samples. The present study was approved by the Human Studies Committee at The Second Affiliated Hospital of Zhengzhou University (Zhengzhou, China). Informed consent was obtained from each patient prior to surgery. A total of 43 pairs of EC samples (all females; age, $54 \pm 16$ years) were obtained from patients; Patients that were treated with chemotherapy or radiotherapy before surgery were excluded. samples were histologically validated for type and grade. These samples were collected from May 2012 to July 2016.

Cell lines and cell culture. Human EC cell lines HHUA and JEC were obtained from the Cell Bank of Type Culture Collection of Chinese Academy of Science (Shanghai, China) and cultured in DMEM (Sigma-Aldrich; Merck KGaA, Darmstadt, Germany) supplemented with $15 \%$ of fetal bovine serum (FBS), $100 \mathrm{U} / \mathrm{ml}$ of penicillin and $100 \mu \mathrm{g} / \mathrm{ml}$ of streptomycin (all from Gibco; Thermo Fisher Scientific, Inc., Waltham, MA, USA). Cells were incubated at $37^{\circ} \mathrm{C}$ in a humidified atmosphere of $5 \% \mathrm{CO}_{2}$. To inhibit the PI3K/AKT signaling pathway, $50 \mu \mathrm{M}$ LY294002 (cat. no. 9901; Cell Signaling Technology, Inc., Danvers, MA, USA) was added to the cultured cells for 24 or $48 \mathrm{~h}$ at $37^{\circ} \mathrm{C}$ according to the manufacturer's protocol.

Cell transfection. miR-494-3p mimics (5'-UGAAACAUACAC GGGAAACCUC-3'), inhibitors (5'-GAGGUUUCCCGUGUA UGUUUCA-3') and negative controls (NCs; 5'-ACAUCUGCG UAAGAUUCGAGUCUA-3') were synthetized by Invitrogen (Thermo Fisher Scientific, Inc.). For PTEN overexpressioin, the coding sequence of PTEN was amplified by PCR and constructed into the pcDNA3 vector (Invitrogen; Thermo Fisher Scientific, Inc.) between EcoRI and XhoI. All transfections were performed using Lipofectamine ${ }^{\circledR} 2000$ (Invitrogen; Thermo Fisher Scientific, Inc.) according to the manufacturer's protocol, at a concentration of $50 \mathrm{nM}$ for miRNAs and $1 \mu \mathrm{g}$ for pcDNA3-PTEN. After $48 \mathrm{~h}$ at $37^{\circ} \mathrm{C}$, the transfection efficiency was validated using qRT-PCR as described below.

In vivo nude mouse xenograft assay. All animal experiments were approved by the Ethics Committee of The Second Affiliated Hospital of Zhengzhou University. Female BALB/c nude mice (4 mice per group; weight, 20.00 $\pm 1.92 \mathrm{~g}$ ), 4-6-weeks-old were obtained from Beijing Vital River Laboratories Animal Technology Co., Ltd. (Beijing, China) and were routinely housed in light-(12 h dark/12 h light) and temperature-controlled rooms $\left(23^{\circ} \mathrm{C}\right)$. These mice were given free access to sterile food and water during the experiment process. HHUA cells $\left(1 \times 10^{7}\right)$ were transfected with miR-494-3p inhibitors or controls resuspended in $200 \mu \mathrm{l}$ FBS-free culture medium and subcutaneously injected into the right flanks of mice. miR-494-3p inhibitors or controls were injected into the formed tumor tissues every three days for 42 days. The tumor volume and weight were determined routinely following inoculation using direct measurement and calculated using the formula (length $\mathrm{x}$ width ${ }^{2}$ )/2. The mice were then sacrificed; tumor tissues were obtained and tumor weights were determined.

Cell proliferation. Cell proliferation was examined using a Cell Counting Kit-8 (CCK8) assay (R\&D Systems, Inc., Minneapolis, MN, USA) according to the manufacturer's protocols. Proliferation was determined through measuring absorbance at $450 \mathrm{~nm}$ using an ELx808 absorbance reader (BioTek Instruments, Inc., Winooski, VT, USA). And a colony formation assay was also conducted. In brief, 1,000 HHUA or JEC cells were seeded into 6 -well plates and cultured for 14 days in DMEM medium at $37^{\circ} \mathrm{C}$. Then the colonies were fixed with paraformaldehyde for $30 \mathrm{~min}$ at $25^{\circ} \mathrm{C}$ and stained with $0.1 \%$ crystal violet for $30 \mathrm{~min}$ at $25^{\circ} \mathrm{C}$. Colony numbers were manually counted.

Transwell assay. To examine cell migration, cells were plated onto a 24-well Transwell chamber (Corning Incorporated, Corning, NY, USA). A total of $2 \times 10^{4}$ cells were diluted in serum-free medium at $24 \mathrm{~h}$ post-transfection and subsequently inoculated onto the upper chamber. A total of $600 \mu \mathrm{l}$ Dulbecco's modified Eagle's medium (Sigma-Aldrich; Merck KGaA) with 10\% FBS (Gibco; Thermo Fisher Scientific, Inc.) was added to the lower chamber at $37^{\circ} \mathrm{C}$. Non-migratory cells were removed using a cotton swab following overnight incubation, while the migratory cells in the lower chamber were fixed with paraformaldehyde for $30 \mathrm{~min}$ at $25^{\circ} \mathrm{C}$ and stained with $0.1 \%$ crystal violet for $30 \mathrm{~min}$ at $25^{\circ} \mathrm{C}$. A cell invasion assay was performed in a similar manner, but Matrigel (Collaborative Research, Bedford, MA, USA) was added to the upper chamber. Finally, the number of migrated and invasive cells were observed and counted using an optical microscope (magnification x200, Nikon Corporation, Tokyo, Japan). Three random fields were counted per group.

Reverse transcription-quantitative polymerase chain reaction $(R T-q P C R)$. Total RNA from tissues or cells was extracted using TRIzol ${ }^{\circledR}$ reagent (Invitrogen; Thermo Fisher Scientific, Inc.) according to the manufacturer's protocols. RNA $(0.5 \mu \mathrm{g})$ was reverse transcribed using a PrimeScript RT Kit (Takara Bio, Inc., Otsu, Japan) according to the manufacturer's protocols. Then, the transcripts were analyzed on an ABI 7300 qPCR system (Applied Biosystem; Thermo Fisher Scientific, Inc.) using specific primers synthesized by Beijing Sunbiotech Co., Ltd. (Beijing, China) using the TaqMan ${ }^{\text {TM }}$ MicroRNA Assay kit (Applied Biosystems; Thermo Fisher Scientific, Inc.) for miRNAs and the Fast SYBR ${ }^{\mathrm{TM}}$-Green Master Mix (Applied Biosystems; Thermo Fisher Scientific Inc.) for mRNAs. The thermocycling conditions were as follows: Denaturation at 
$95^{\circ} \mathrm{C}$ for $10 \mathrm{~min}$; followed by 40 cycles of denaturation at $95^{\circ} \mathrm{C}$ for $15 \mathrm{sec}$ and elongation at $60^{\circ} \mathrm{C}$ for $1 \mathrm{~min}$. Three repearts were performed. Relative expression was calculated and normalized to endogenous $\beta$-actin (ACTB) or U6. Relative expression levels were evaluated via the $2^{-\Delta \Delta \mathrm{Cq}}$ method (17). Primer sequences were as follows: miR-494-3p, forward, 5'-AACGAGACGACGACAGAC-3' and reverse, 5'-TGAAAC ATACACGGGAAACCTC-3'; U6 forward, 5'-AACGAGACG ACGACAGAC-3' and reverse, 5'-GCAAATTCGTGAAGC GTTCCATA-3'; PTEN forward, 5'-TCCCAGACATGACAG CCATC-3' and reverse, 5'-TGCTTTGAATCCAAAAACCTT ACT-3', and ACTB forward, 5'-CGGCGCCCTATAAAA CCCA-3' and reverse, 5'-GAGGCGTACAGGGATAGCAC-3'.

Western blotting. Total protein was isolated from cultured HHUA and JEC cells using radioimmunoprecipitation assay buffer (Thermo Fisher Scientific, Inc), and the supernatant was collected via centrifugation at $13,282 \times \mathrm{g}$ for $10 \mathrm{~min}$ at $4^{\circ} \mathrm{C}$. Protein concentration was evaluated using a Pierce BCA Protein Assay kit (Pierce; Thermo Fisher Scientific, Inc.). Subsequently, the extracted protein was mixed with loading buffer and boiled at $100^{\circ} \mathrm{C}$ for $5 \mathrm{~min}$. Total protein $(30 \mu \mathrm{g})$ was separated using 10\% SDS-PAGE gels and transferred onto polyvinylidene fluoride membranes (EMD Milipore, Billerica, MA, USA). Subsequently, the membranes were blocked with $5 \%(\mathrm{w} / \mathrm{v})$ non-fat milk for $2 \mathrm{~h}$ at $25^{\circ} \mathrm{C}$ and incubated with antibodies against PTEN (1:2,000; cat. no. 9188), AKT (1:2,000; cat. no. 4691), phosphorylated-AKT (1:2,000; cat. no. 4060), PI3K (1:,2,000; cat. no. 4249), p-PI3K (1:2,000; cat. no. 4228), BCL2 (1:2,000; cat. no. 2872), caspase-3 (1:2,000; cat. no. 9664) or GAPDH (1:2,000; cat. no. 5174) (all from Cell Signaling Technology, Inc.) for $2 \mathrm{~h}$ at $25^{\circ} \mathrm{C}$. The membranes were then probed with a horseradish peroxidase-conjugated secondary antibody (1:5,000; cat. no. ab7090; Abcam, Cambridge, UK) at $25^{\circ} \mathrm{C}$ for $1 \mathrm{~h}$, and signals were visualized using an enhanced chemiluminescence kit (Beyotime Institute of Biotechnology, Beijing, China) according to manufacturer's protocol.

Luciferase reporter assay. The potential binding site for miR-494-3p in PTEN 3'-UTR region was predicted using the TargetScan7 tool (www.targetscan.org/vert_71/). Cells were seeded on a 6 -well-plate at a density of $1 \times 10^{6}$ cells/well and transfected with $50 \mathrm{nM}$ miR-494-3p mimics or negative controls, $100 \mathrm{ng}$ pGL3-PTEN 3'-UTR-Wild type or Mutant vector and $1 \mathrm{ng}$ pRL-TK Renilla luciferase plasmid (Promega Corporation, Madison, WI, USA) according to the manufacturer's protocols (Lipofectamine RNAiMAX, Invitrogen; Thermo Fisher Scientific, Inc.). After 48 h, luciferase assays were performed using the dual-luciferase reporter assay system (Promega Corporation) according to the manufacturer's protocols. Luminescent signals were quantified with a luminometer (Glomax, Promega Corporation), and the value of firefly luciferase was normalized to that of Renilla luciferase.

Statistical analysis. Statistical analysis was performed using SPSS 17.0 (SPSS Inc., Chicago, IL, USA). The differences among groups, in at least three separate experiments, were analyzed using a Student's t-test or one-way analysis of variance followed by Dunnett's multiple comparison test, as appropriate. The samples were divided into miR-494-3p low
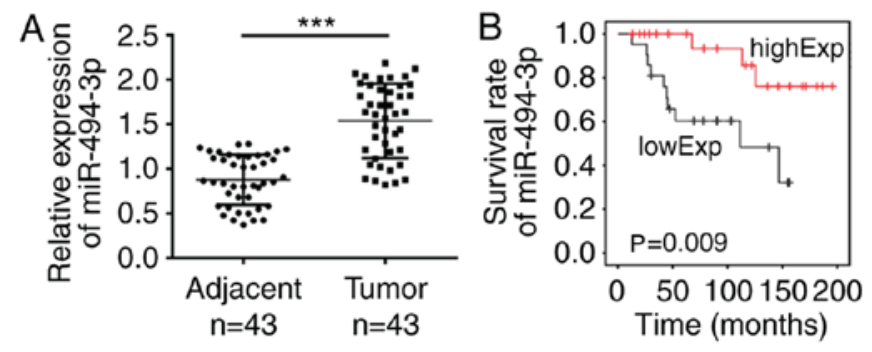

Figure 1. miR-494-3p is upregulated in EC tissues. (A) Reverse transcription-quantitative polymerase chain reaction revealed that the expression levels of miR-494-3p in EC tissues were significantly upregulated compared with adjacent normal tissues. ${ }^{* * *} \mathrm{P}<0.001$ vs. adjacent normal tissues. (B) Patients with EC and increased miR-494-3p expression had lower overall survival rates as indicated by Kaplan-Meier analysis and a log-rank test. EC, endometrial cancer; miR, microRNA.

and high expression groups according to the median value of miR-494-3p. Then Kaplan-Meier survival analysis and log-rank test were used for survival evaluation. Spearman's rank correlation analysis was performed to analyze correlation between miR-494-3p and PTEN expression levels. P<0.05 was considered to indicate a statistically significant difference.

\section{Results}

miR-494-3p is upregulated in EC tissues. To investigate the function of miR-494-3p in EC, 43 EC samples were employed and the expression of miR-494-3p was determined by RT-qPCR in the present study. The results indicated that miR-494-3p was significantly upregulated in EC tissues compared with adjacent normal tissues (Fig. 1A). Then, these tissues were divided into low- or high-expression subgroups according to miR-494-3p expression, followed by Kaplan-Meier survival analysis. As presented in Fig. 1B, increased expression of miR-494-3p in patients with EC indicated poorer prognosis.

Overexpression of miR-494-3p promotes EC cell proliferation, migration and invasion. To further determine the effects of miR-494-3p on EC cells, miR-494-3p was overexpressed in EC cell lines (HHUA and JEC; Fig. 2A). Then, CCK8 and colony formation assays were performed using HHUA and JEC cells transfected with miR-494-3p mimics or controls. The results revealed that overexpression of miR-494-3p significantly promoted cellular proliferation and increased the number of the colonies compared with the control group (Fig. 2B and C). In addition, the effects of miR-494-3p on cellular apoptosis were evaluated by analysing the protein expression levels of caspase-3 and B-cell lymphoma-2 (Bcl-2). The results revealed that overexpression of miR-494-3p did not affect caspase-3 and Bcl-2 expression (data not shown), indicating that apoptosis was not affected by miR-494-3p. Tumor metastasis is one of the main causes of tumor malignancy (18). The effects of miR-494-3p on tumor cell metastasis were investigated using a Transwell assay. As presented, overexpression of miR-494-3p significantly promoted the migration and invasion of HHUA and JEC cells compared with the control group (Fig. 2D and E). Furthermore, the effects of miR-494-3p inhibition were evaluated by CCK8 and Transwell assays. miR-494-3p 

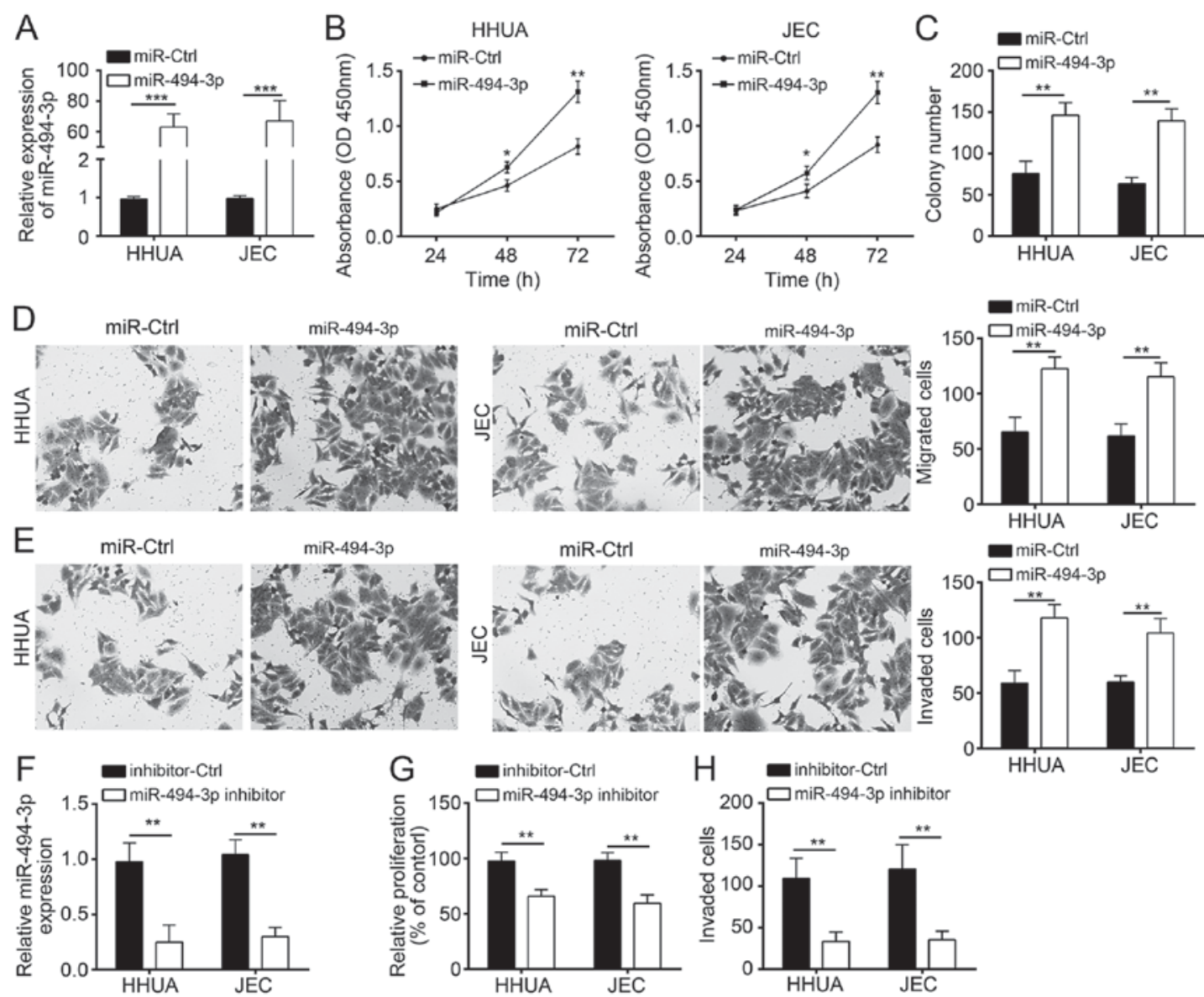

Figure 2. Overexpression of miR-494-3p promotes the proliferation, migration and invasion of endometrial cancer cells. (A) Following miR-494-3p transfection, endometrial adenocarcinoma cell lines exhibited significantly upregulated expression of miR-494-3p. (B and C) Cellular proliferation was evaluated by CCK8 and colony formation assays in HHUA and JEC cells transfected with miR-494-3p mimics or controls. (D and E) Following miR-494-3p transfection, endometrial adenocarcinoma cell lines exhibited increased migration and invasive abilities as revealed by Transwell assays (magnification, $\mathrm{x} 200$ ). (F) Relative expression of miR-494-3p was determined by reverse transcription-quantitative polymerase chain reaction. (G) Proliferation of HHUA and JEC cells transfected with miR-494-3p inhibitors or controls was evaluated using CCK8 assays. The optical density value was measured $72 \mathrm{~h}$ post-transfection. (H) Transwell invasion assay indicated that miR-494-3p inhibitor suppressed tumor cell invasion. ${ }^{*} \mathrm{P}<0.05,{ }^{* *} \mathrm{P}<0.01$ and ${ }^{* * *} \mathrm{P}<0.001$ vs. miR-Ctrl or inhibitor-Ctrl. Ctrl, control; CCK8, Cell Counting Kit-8; miR, microRNA.

expression levels were significantly inhibited following transfection with miR-494-3p inhibitors (Fig. 2F). In addition, the results demonstrated that miR-494-3p inhibitor significantly suppressed the proliferation and invasion of HHUA and JEC cells compared with the control (Fig. 2G and H).

Inhibition of miR-494-3p suppresses tumor growth in vivo. To further investigate the physiological function of miR-494-3p in vivo, a xenograft experiment was performed using HHUA cells. miR-494-3p-silenced or control HHUA cells were injected into nude mice. At indicative time points following injection, the tumor volumes were measured; miR-494-3p knockdown significantly delayed tumor growth in vivo compared with the control (Fig. 3A). In addition, the tumor weights were determined at the endpoints of experiments. As presented in Fig. 3B, miR-494-3p knockdown resulted in significantly reduced tumor weight compared with the control.

miR-494-3p activates the PI3K/AKT signaling pathway by targeting PTEN. The miR-494-3p-regulated molecular mechanisms associated with EC were investigated. According to bioinformatic prediction, PTEN was identified to be a potential target gene of miR-494-3p. There were two conserved potential binding sites in the 3'-UTR of PTEN mRNA (Fig. 4A); then, luciferase reporter assays were conducted. As presented in Fig. 4B, overexpression of miR-494-3p significantly inhibited luciferase activity in HHUA and JEC cells compared with the control. Furthermore, the present study reported that overexpression of miR-494-3p significantly downregulated the mRNA expression levels of PTEN in HHUA and JEC cells compared with the control (Fig. 4C). Then, the expression levels of miR-494-3p and PTEN in EC tissues were evaluated. The results of RT-qPCR indicated that there was an inverse correlation between the expression levels of miR-494-3p and PTEN in EC tissues (Fig. 4D). A recent study revealed that the downstream signaling of PTEN comprised the PI3K/AKT pathway (8), which was widely involved in various human cancers, such as EC $(19,20)$. Furthermore, the effects of miR-494-3p on PTEN protein expression and PI3K/AKT activation were evaluated using western blot analysis. Overexpression of miR-494-3p notably downregulated the protein expression levels of PTEN, and upregulated the phosphorylation of PI3K and AKT (Fig. 4E). In summary, 

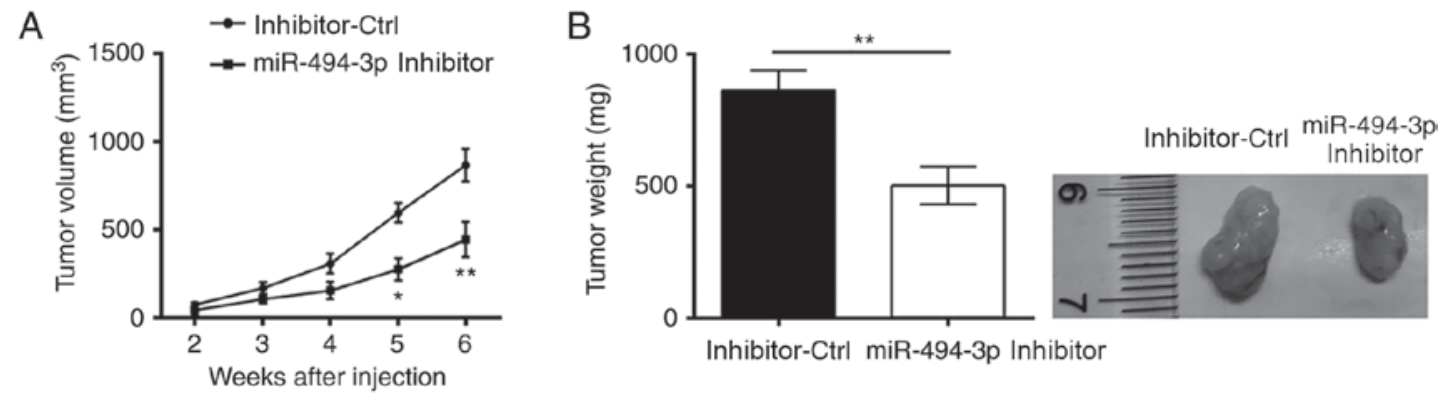

Figure 3. Inhibition of miR-494-3p suppresses tumor growth in vivo. (A) Inhibition of miR-494-3p suppressed tumor growth in vivo; tumor volumes were measured at indicative time points. $n=5$ for each group. (B) Tumor weights were measured 6 weeks in total following injection. Representative images of tumor in each group were presented. ${ }^{*} \mathrm{P}<0.05$ and ${ }^{* *} \mathrm{P}<0.01$ vs. Inhibitor-Ctrl. Ctrl, control; miR, microRNA.

A

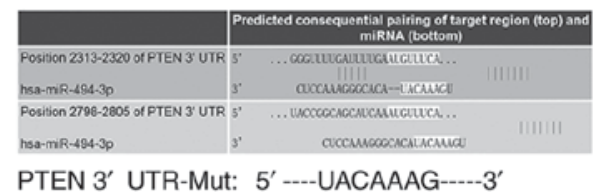

B

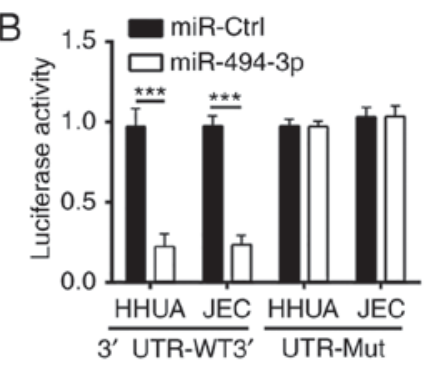

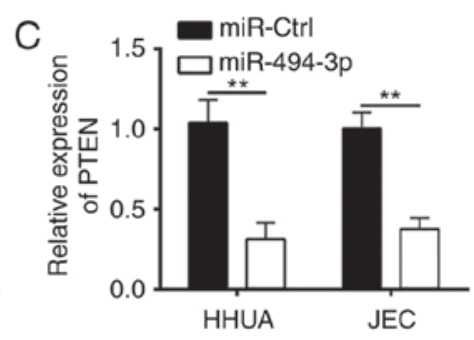
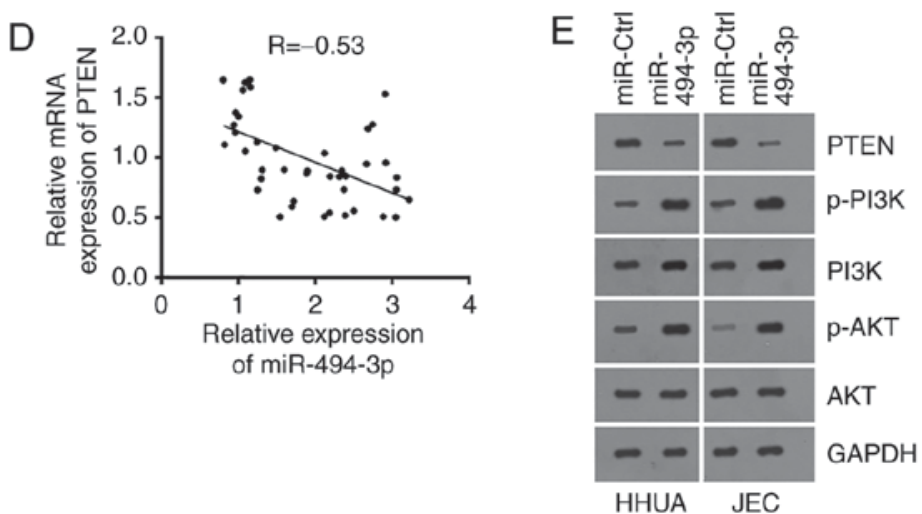

Figure 4. miR-494-3p activates the PI3K/AKT pathway by targeting PTEN. (A) A total of two potential binding sites of miR-494-3p were identified in the 3'-UTR region of PTEN mRNA. (B) Overexpression of miR-494-3p significantly inhibited the luciferase activity in HHUA and JEC cells, while mutation of the complementary sequence in the 3'-UTR region of PTEN mRNA abrogated this inhibitory effect. (C) Overexpression of miR-494-3p downregulated the mRNA expression levels of PTEN in HHUA and JEC cells. (D) Expression levels of miR-494-3p and PTEN were inversely correlated in endometrial cancer tissues. (E) Overexpression of miR-494-3p activated PI3K/AKT pathway and downregulated the protein expression levels of $\mathrm{PTEN}$. ${ }^{* *} \mathrm{P}<0.01$ and ${ }^{* * *} \mathrm{P}<0.001$. Ctrl, control; miR, microRNA; PI3K/AKT, phosphoinositide 3-kinase/protein kinase B; PTEN, phosphatase and tensin homolog; UTR, untranslated region.

the aforementioned data indicated that miR-494-3p activated the PI3K/AKT pathway by inhibiting PTEN in EC cells.

Restoration of PTEN or inhibition of the PI3K/AKT pathway abolishes miR-494-3p-mediated effects on EC cells. To determine whether miR-494-3p affects EC cell proliferation, migration and invasion via the PTEN/PI3K/AKT pathway, the protein expression of PTEN was restored or the PI3K/AKT pathway was inhibited using a specific inhibitor (LY294002) in miR-494-3p-overexpressed HHUA and JEC cells (Fig. 5A). Then, CCK8 and colony formation assays were performed to evaluate cellular proliferation. As presented in Fig. 5B and C, overexpression of miR-494-3p signficantly promoted cell proliferation compared with the control; however, restoration of PTEN or inhibition of the PI3K/AKT pathway abrogated the effects of miR-494-3p overexpression on EC cells. Similarly, overexpression of miR-494-3p significantly promoted cellular migration and invasion compared with the control; however restoration of PTEN or inhibition of the PI3K/AKT pathway in miR-494-3p-overexpressed HHUA and JEC cells reversed these effects (Fig. 5D and E). In summary, the results in the present study demonstrated that miR-494-3p promoted the progression of EC via the PTEN/PI3K/AKT signaling pathway.

\section{Discussion}

MiRNAs can regulate the development and progression of EC (21); however, the functions of numerous miRNAs in EC have not been determined. In the present study, the expression levels of miR-494-3p were significantly upregulated in EC tissues compared with adjacent normal tissues. Additionally, the expression of miR-494-3p was positively associated with 

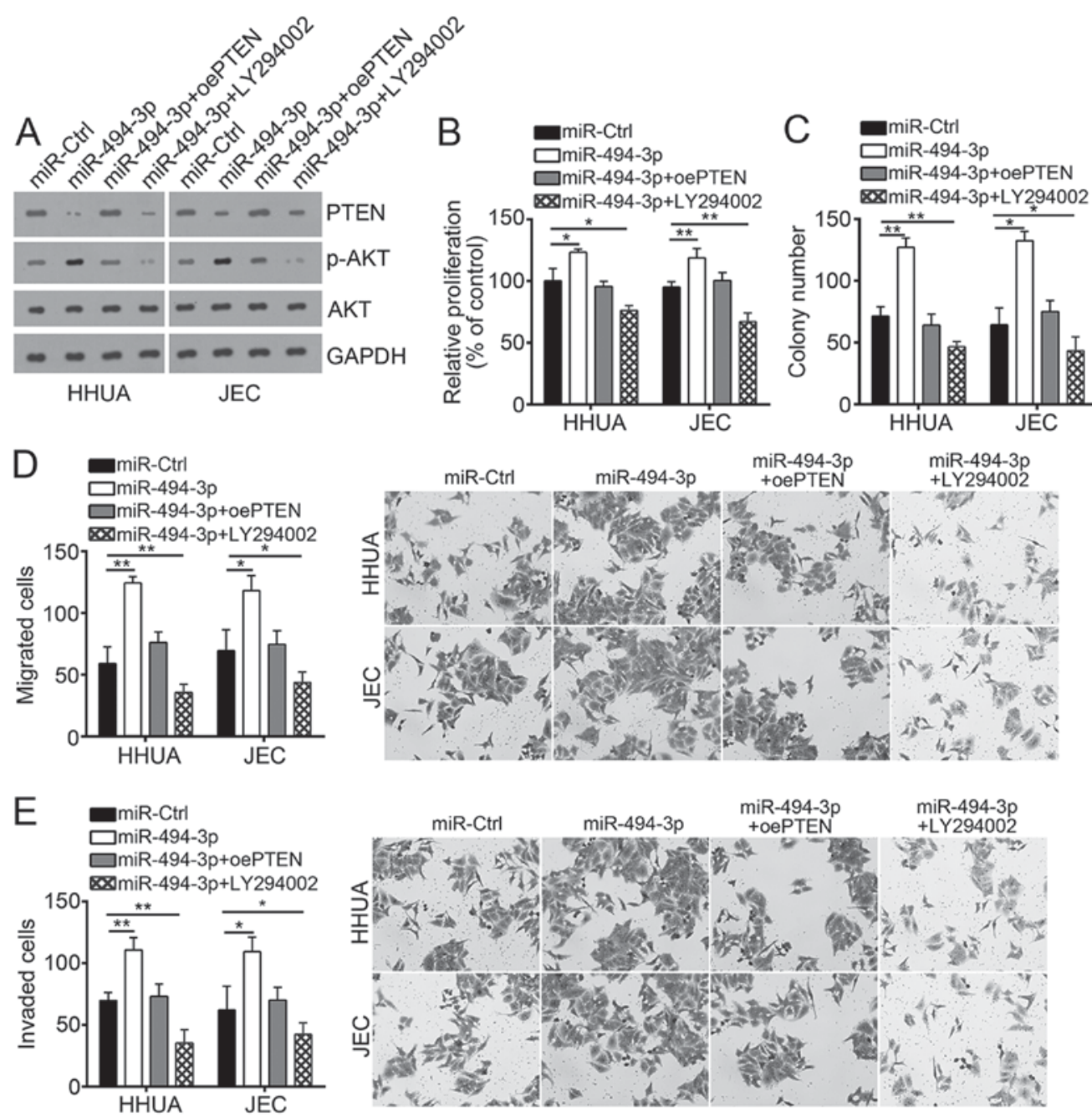

Figure 5. Restoration of PTEN or inhibition of the PI3K/AKT pathway abolishes miR-494-3p-mediated effects on endometrial cancer cells. (A) PTEN expression with an oe plasmid was restored by ectopic expression and the PI3K/AKT pathway was suppressed by the addition of LY294002. (B and C) Restoration of PTEN or inhibition of the PI3K/AKT pathway suppressed the proliferation of HHUA and JEC cells overexpressing miR-494-3p. (D and E) Restoration of PTEN or inhibition of PI3K/AKT pathway suppressed the migration and invasion of HHUA and JEC cells overexpressing miR-494-3p. ${ }^{*} \mathrm{P}<0.05$ and ${ }^{* *} \mathrm{P}<0.01$. Ctrl, control; miR, microRNA; PI3K/AKT, phosphoinositide 3-kinase/protein kinase B; PTEN, phosphatase and tensin homolog; oe, overexpression.

the poor prognosis of patients with EC. Therefore, miR-494-3p may serve as an oncogene in EC, consequently promoting tumor progression.

Numerous studies demonstrated that miR-494-3p was an essential regulator in numerous types of cancer. For example, a recent study indicated that miR-494-3p promoted the development of lung cancer (14). Liu et al (22) reported that miR-494 promoted cell proliferation, migration and invasion, and increased sorafenib resistance in hepatocellular carcinoma by targeting PTEN. Li et al (15) revealed that miR-494-3p regulated cellular proliferation, invasion, migration, and apoptosis via PTEN/AKT signaling in human glioblastoma cells. Additionally, some studies have indicated that miR-494 served as a tumor suppressor in certain types of cancer. For instance, miR-494-3p could induce cellular senescence and enhance radiosensitivity of oral squamous carcinoma cells (23). Shen et al (16) demonstrated that miRNA-494-3p targeted $\mathrm{C}-\mathrm{X}-\mathrm{C}$ chemokine receptor type 4 (CXCR4) to suppress the proliferation, invasion and migration of prostate cancer cells. These reported contrary functions of miR-494-3p that may be due to different target genes in various types of cancer. In the present study, overexpression of miR-494-3p significantly promoted cellular proliferation in EC in vitro and in vivo. In addition, overexpressed miR-494-3p induced cellular migration and invasion in vitro. The findings of the present study indicated an oncogenic role of miR-494-3p in EC.

Furthermore, previous studies revealed that PTEN could regulate a variety of biological processes, including the cell cycle, apoptosis, migration and invasion by inhibiting the PI3K/AKT signaling pathway $(24,25)$. PTEN mutations, deletions or silencing by promoter hypermethylation often results in the tumorigenesis of several cancers, such as EC (26). A previous study indicated that PTEN was mutated in $83 \%$ of EC tissues (27). Downregulation of PTEN was associated with activation of the PI3K/AKT pathway, consequently promoting tumor development and progression (28). For example, E3 ubiquitin-protein ligase regulated PTEN/PI3K/AKT signaling to promote the cell growth and migration of hepatocellular carcinoma cells (29). Liu et al (30) reported that Sal-like protein 4 suppressed PTEN expression to promote glioma cell proliferation via the PI3K/AKT pathway. Another study revealed that miRNA-1297 contributed to tumor growth 
of human breast cancer by targeting the PTEN/PI3K/AKT signaling pathway (28). In addition, miRNA-92a promoted epithelial-mesenchymal transition via the PTEN/PI3K/AKT pathway in metastatic non-small cell lung cancer (18). In the present study, miR-494-3p directly targeted PTEN and downregulated its expression. Furthermore, restoration of PTEN and inhibition of the PI3K/AKT pathway inhibited the proliferation, migration and invasion of EC cells overexpressing miR-494-3p, which indicated that miR-494-3p regulated the progression of EC in a PTEN/PI3K/AKT-dependent manner.

In summary, the findings of the present study revealed the essentialrole of miR-494-3pandits functionalmechanismsinEC. The results suggested that the miR-494-3p/PTEN/PI3K/AKT axis may be a promising therapeutic target for the treatment of EC.

\section{Acknowledgements}

Not applicable.

\section{Funding}

The present study was supported by Science and Technology Department of Sichuan Province (grant no. 2018SZ0264).

\section{Availability of data and materials}

All data generated or analyzed during this study are included in this published article.

\section{Authors' contributions}

LZ initiated, designed this work, analyzed and interpreted the results. $\mathrm{LZ}$ wrote this manuscript. XW, TW, WZ and XZ performed the experiments. All authors read and approved the final manuscript.

\section{Ethics approval and consent to participate}

For the use of human samples, the protocol for the present study was approved by the Institutional Ethics Committee of the Second Affiliated Hospital of Zhengzhou University (Zhengzhou, China) and all enrolled patients signed a written informed consent document. All animal experiments were approved by the Ethics Committee of the Second Affiliated Hospital of Zhengzhou University.

\section{Patient consent for publication}

Not applicable.

\section{Competing interests}

The authors declare that they have no competing interests.

\section{References}

1. Banno K, Yanokura M, Iida M, Masuda K and Aoki D: Carcinogenic mechanisms of endometrial cancer: Involvement of genetics and epigenetics. J Obstet Gynaecol Res 40: 1957-1967, 2014.
2. Yeramian A, Moreno-Bueno G, Dolcet X, Catasus L, Abal M, Colas E, Reventos J, Palacios J, Prat J and Matias-Guiu X: Endometrial carcinoma: Molecular alterations involved in tumor development and progression. Oncogene 32: 403-413, 2013.

3. Eritja N, Chen BJ, Rodriguez-Barrueco R, Santacana M, Gatius S, Vidal A, Martí MD, Ponce J, Bergadà L, Yeramian A, et al: Autophagy orchestrates adaptive responses to targeted therapy in endometrial cancer. Autophagy 13: 608-624, 2017.

4. Colombo N, Creutzberg C, Amant F, Bosse T, González-Martín A, Ledermann J, Marth C, Nout R, Querleu D, Mirza MR, et al: ESMO-ESGO-ESTRO consensus conference on endometrial cancer: Diagnosis, treatment and follow-up. Ann Oncol 27: 16-41, 2016

5. Ramon LA, Braza-Boils A, Gilabert J, Chirivella M, España F, Estellés A and Gilabert-Estellés J: microRNAs related to angiogenesis are dysregulated in endometrioid endometrial cancer. Hum Reprod 27: 3036-3045, 2012.

6. Ambros V: The functions of animal microRNAs. Nature 431: 350-355, 2004.

7. Bartel DP: MicroRNAs: Genomics, biogenesis, mechanism, and function. Cell 116: 281-297, 2004.

8. Zhao C, Sun W, Zhang P, Ling S, Li Y, Zhao D, Peng J, Wang A, Li Q, Song J, et al: miR-214 promotes osteoclastogenesis by targeting Pten/PI3k/Akt pathway. RNA Biol 12: 343-353, 2015.

9. Madhavan B, Yue SJ, Galli U, Rana S, Gross W, Müller M, Giese NA, Kalthoff H, Becker T, Büchler MW and Zöller M: Combined evaluation of a panel of protein and miRNA serum-exosome biomarkers for pancreatic cancer diagnosis increases sensitivity and specificity. Int J Cancer 136: 2616-2627, 2015.

10. Li Z, Yu X, Shen J and Jiang Y: MicroRNA dysregulation in uveal melanoma: A new player enters the game. Oncotarget 6: 4562-4568, 2015

11. Zeng B, Shi W and Tan G: miR-199a/b-3p inhibits gastric cancer cell proliferation via down-regulating PAK4/MEK/ERK signaling pathway. BMC Cancer 18: 34, 2018.

12. Li S, Luo C, Zhou J and Zhang Y: MicroRNA-34a directly targets high-mobility group box 1 and inhibits the cancer cell proliferation, migration and invasion in cutaneous squamous cell carcinoma. Exp Ther Med 14: 5611-5618, 2017.

13. Zhao X, Zhu D, Lu C, Yan D, Li L and Chen Z: MicroRNA-126 inhibits the migration and invasion of endometrial cancer cells by targeting insulin receptor substrate 1. Oncol Lett 11: 1207-1212, 2016.

14. Faversani A, Amatori S, Augello C, Colombo F, Porretti L, Fanelli M, Ferrero S, Palleschi A, Pelicci PG, Belloni E, et al: miR-494-3p is a novel tumor driver of lung carcinogenesis. Oncotarget 8: 7231-7247, 2017.

15. Li XT, Wang HZ, Wu ZW, Yang TQ, Zhao ZH, Chen GL, Xie XS, Li B, Wei YX, Huang YL, et al: miR-494-3p regulates cellular proliferation, invasion, migration and apoptosis by PTEN/AKT signaling in human glioblastoma cells. Cell Mol Neurobiol 35: 679-687, 2015.

16. Shen PF, Chen XQ, Liao YC, Chen N, Zhou Q, Wei Q, Li X, Wang J and Zeng H: MicroRNA-494-3p targets CXCR4 to suppress the proliferation, invasion, and migration of prostate cancer. Prostate 74: 756-767, 2014.

17. Livak KJ and Schmittgen TD: Analysis of relative gene expression data using real-time quantitative PCR and the 2(-Delta Delta C(T)) method. Methods 25: 402-408, 2001.

18. Lu CJ, Shan ZX, Hong J and Yang L: MicroRNA-92a promotes epithelial-mesenchymal transition through activation of PTEN/PI3K/AKT signaling pathway in non-small cell lung cancer metastasis. Int J Oncol 51: 235-244, 2017.

19. Sun K, Wang S, He J, Xie Y, He Y, Wang Z and Qin L: NCOA5 promotes proliferation, migration and invasion of colorectal cancer cells via activation of PI3K/AKT pathway. Oncotarget 8: 107932-107946, 2017.

20. Zhang S, Wang M, Li Q and Zhu P: miR-101 reduces cell proliferation and invasion and enhances apoptosis in endometrial cancer via regulating PI3K/Akt/mTOR. Cancer Biomark 21: 179-186, 2017.

21. Li L, Qu YW and Li YP: Over-expression of miR-1271 inhibits endometrial cancer cells proliferation and induces cell apoptosis by targeting CDK1. Eur Rev Med Pharmacol Sci 21: 2816-2822, 2017.

22. Liu K, Liu S, Zhang W, Jia B, Tan L, Jin Z and Liu Y: miR-494 promotes cell proliferation, migration and invasion, and increased sorafenib resistance in hepatocellular carcinoma by targeting PTEN. Oncol Rep 34: 1003-1010, 2015. 
23. Weng JH, Yu CC, Lee YC, Lin CW, Chang WW and Kuo YL: miR-494-3p induces cellular senescence and enhances radiosensitivity in human oral squamous carcinoma cells. Int J Mol Sci 17: E1092, 2016

24. Qiu HF, Li J, Clark LH, Jackson AL, Zhang L, Guo H, Kilgore JE, Gehrig PA, Zhou C and Bae-Jump VL: JQ1 suppresses tumor growth via PTEN/PI3K/AKT pathway in endometrial cancer. Oncotarget 7: 66809-66821, 2016

25. Zhang J, Yang Y, Zhang Z, He Y, Liu Z, Yu Y, Wu S, Cai B and Feng Y: Gankyrin plays an essential role in estrogen-driven and GPR30-mediated endometrial carcinoma cell proliferation via the PTEN/PI3K/AKT signaling pathway.CancerLett339:279-287,2013.

26. Cancer Genome Atlas Research Network, Kandoth C, Schultz N, Cherniack AD, Akbani R, Liu Y, Shen H, Robertson AG, Pashtan I, Shen R, et al: Integrated genomic characterization of endometrial carcinoma. Nature 497: 67-73, 2013.
27. Mutter GL, Lin MC, Fitzgerald JT, Kum JB, Baak JP, Lees JA, Weng LP and Eng C: Altered PTEN expression as a diagnostic marker for the earliest endometrial precancers. J Natl Cancer Inst 92: 924-931, 2000.

28. Liu C, Liu ZK, Li X, Tang XJ, He JJ and Lu SY: MicroRNA-1297 contributes to tumor growth of human breast cancer by targeting PTEN/PI3K/AKT signaling. Oncol Rep 38: 2435-2443, 2017.

29. Huang ZJ, Zhu JJ, Yang XY and Biskup E: NEDD4 promotes cell growth and migration via PTEN/PI3K/AKT signaling in hepatocellular carcinoma. Oncol Lett 14: 2649-2656, 2017.

30. Liu CJ, Wu HB, Li YY, Shen L, Yu R, Yin H, Sun T, Sun C, Zhou Y and Du Z: SALL4 suppresses PTEN expression to promote glioma cell proliferation via PI3K/AKT signaling pathway. J Neurooncol 135: 263-272, 2017. 